\title{
Robust, Transparent and Self-healing
}

\section{Superamphiphobic Films}

Min Wen ${ }^{l}$, Jie Zhong ${ }^{1 *}$, Shuangjie Zhao ${ }^{1}$, Tongle Bu ${ }^{l}$, Le Guo ${ }^{1}$, Zhiliang Ku ${ }^{l}$, Yong Peng

1, Fuzhi Huang ${ }^{\text {, }}$ Yi-Bing Cheng ${ }^{1,2}$, Qi Zhang 1,3*

'State Key Laboratory of Advanced Technology for Materials Synthesis and Processing, School of Materials Science and Engineering, Wuhan University of Technology, Wuhan 430070, China

${ }^{2}$ Department of Materials Science and Engineering, Monash University, VIC 3800, Australia

${ }^{3}$ School of Aerospace, Transport and Manufacturing, Cranfield University,

Cranfield, Bedfordshire, MK43 0AL, United Kingdom

*Corresponding author, email: jie.zhong@whut.edu.cn; q.zhang@ cranfield.ac.uk.

KEYWORDS: Superamphiphobic, Superhydrophobic, Transparent, Self-healing, Robust, Spray

\begin{abstract}
The technological implementation of superamphiphobic surfaces has been largely hindered by their stability issues caused by surface abrasion, corrosion, contamination and etc. The contradictory requirement on both decent roughness and robustness is still remain the major challenge for a well-performing superamphiphobic coating. In this work, a simple route of spraying the inks containing designed silica building-blocks are presented to preparing superamphiphobic coatings. The as-made films exhibit excellent super-repellency to various
\end{abstract}

\section{ACS Paragon Plus Environment}


liquids and also enhanced robustness. The static contact angles (SCAs) for water (surface tension $72.1 \mathrm{mN} / \mathrm{m}$ ) and dodecane (surface tension $25.3 \mathrm{mN} / \mathrm{m}$ ) can reach $166 \pm 3^{\circ}$ and $153 \pm 3^{\circ}$, respectively. Through controlling thickness of the films, the optical transmittance of the films (400 nm thick) is close to that of glass. The mechanical properties of the films are significantly strengthened for titania modified silica coatings, which are able to withstand a standard $2 \mathrm{H}$ pencil scratching and sand flow impact. Moreover, efficient decomposition of organic substance attached on the surfaces by photocatalysis has been demonstrated which enables the recovery of superamphiphobic property of the contaminated films. Thus, these unique properties, robustness, transparency and self-healing, etc., combined with the low cost fabrication, make the superamphiphobic coatings promising in various applications.

Inspired by biological species, such as water-repellent plants, ${ }^{1}$ water striders, ${ }^{2}$ nepenthes, 3 butterfly, ${ }^{4}$ dragonfly ${ }^{5}$ and so on, ${ }^{6-8}$ the fine micro-nano structure with super-repellent property could exert tremendous and fascinated applications in self-cleaning, ${ }^{9}$ anti-smudge, ${ }^{10}$ anticorrosion, 11 anti-icing, ${ }^{12}$ heart-lung machines, ${ }^{13}$ and etc. ${ }^{14-15}$ A super-repellent surface can reject bulk liquids being spread on it, which exhibits a large static contact angle (SCAs $>150^{\circ}$ ) and small sliding angles $\left(\mathrm{SAs}<10^{\circ}\right)$. A superhydrophobic surface is super-repellent to water, and a superamphiphobic surface is super-repellent to both water and oil. The main fundamentals of the solid wetting phenomenon, including the Young's model, ${ }^{16}$ the Wenzel's model, ${ }^{17}$ the CassieBaxter's model ${ }^{18}$ and so on, ${ }^{19-21}$ can roughly illustrate the principle of building super-repellent surfaces. Hierarchical structures coated with low surface energy materials can render superhydrophobic surfaces. However, simply roughened surface is not sufficient to create superamphiphobic property because oils have much lower surface tensions than water. In order to build a superamphiphobic surface, a special structure, such as over-hang structure, doubly re- 
entrant structure, ${ }^{22}$ must be developed. So far, there have been many reports claiming that superamphiphobic surfaces were created. ${ }^{22-27}$ However, the superamphiphobic performance of these surfaces varies with the types of oil that could have different and relatively low surface tensions $(\gamma)$, and deteriorates dramatically with extremely low $\gamma$ oils. Very few reports claimed that the surfaces could super-repel the liquids that possess the $\gamma$ as low as $25.3 \mathrm{mN} / \mathrm{m}$ (dodecane) on the non-fabric flat substrates simply by building a special over-hang structure ${ }^{22-}$ ${ }^{27}$ with a complex method, such as, candle soot template, ice template, carbon nanotube template, and etching, etc.

To realize the technological application of superamphiphobic surfaces, three challenges need to be tackled. Firstly, aforementioned micro-nano over-hang structure 22, 28 must be built, which ensures high SCAs for liquids. Many methods, 23, 27, 29-39 such as etching, photolithographic, laser patterning, electrospinning, electrodeposition, templating, spray-coating, etc., have been utilized to construct superamphiphobic films. It is worth to note that using a low-cost, simple, efficient and facile process is essentially required for large scale production of the superamphiphobic films. Spraying coating, as a widely used film deposition technique in industry, could meet these requirements, if a specially-designed particles suspension deposition procedure is used. Different sized $\mathrm{TiO}_{2}$ nanoparticles mixture $(\sim 60-200 \mathrm{~nm}$ and $\sim 21 \mathrm{~nm}),{ }^{38}$ raspberry-like polymer particles, 40 silica nanotubes, ${ }^{41}$ have been used to preparing over-hang structures. Secondly, the mechanical robustness for the films must be enhanced, which needs to withstand possible scratches and abrasions. In spite of the obvious advantages of spraying technology that can easily form delicate micro-nano structures via layer by layer construction, the adhesions between the film and the substrate and also inter-particles are particularly weak if without treatment. To date, the fluorinated polymers or other organic monomers are used as the binders to improve the 
mechanical property, 25-26, 42-43 which are not suitable for the long-term outdoor applications due to the organic aging. Inorganic adhesives ${ }^{44}$ are rarely used due to their high energy barrier of covalent bonding; however, they are less susceptible to the property degradation in the outdoor environment. Finally, the superamphiphobic films must have a long-term stability against atomospheric contamination. Since the superamphiphobic surfaces are theoretically repellent only to bulk liquids, tiny organic pollutants contained in the floating fog and smoke could attach to the superamphiphobic surfaces, therefore altering the surface energy and gradually worsening superrepelling performance. Applying photoactive agent, ${ }^{45-48}$ such as $\mathrm{TiO}_{2}$, to the surfaces could improve the stability against pollutants through photo decomposition of organic contaminants, which provides self-healing ability to superamphiphobic surfaces.

Herein, this paper presents a simple and facile method to prepare the superaphiphobic films by spray deposition of pre-designed silica building blocks. ${ }^{49}$ By adjusting the degree of agglomeration of the nano-SiO 2 particles, the over-hang structure of nanoparticles was obtained and the resultant films show the SCAs as high as $153^{\circ}$ for dodecane and $166^{\circ}$ for water after the films were fluorinated. The thickness of the films can be altered to maintain the superamphipbobic property and simultaneously high transparency to the extent of over $89 \%$. The robustness of the film is enhanced to $2 \mathrm{H}$ hardness pencil scratching ((ASTM) D3363) after incorporation of titania as the bolting crystal for the silica matrix. Moreover, we also demonstrate the interesting self-healing phenomenon of the contaminated superamphiphobic surfaces after a period of illumination under 1 sun solar simulator. The enhanced mechanical property, high transparency and self-healing ability of superamphiphobic films could be attractive in many applications in the future.

\section{RESULTS AND DISCUSSION}




\section{Film building-blocks and Superamphiphobic Coatings}

For the porous films, the static contact angle, $\theta^{\text {app }}\left(\theta^{\text {app }}\right.$ is the apparent contact angle on the surface) can be explained by the Cassie model, whose equation has recently been rewritten as follows: 50$$
\cos \theta={ }_{f} \cos \theta-1+(1)
$$

where $f$ is the fraction of the projected area of the solid surface in contact with the liquid, $\mathrm{rf}$ the roughness of the solid surface that is in contact with water, and $\theta$ the equilibrium contact angle on a smooth surface. To produce a superamphiphobic film with over-hang and continuous pore structure, one of the key issues is to produce a small $\mathrm{f}$ surface ${ }^{50}$. It was found that the nanoparticles with a certain degree of agglomeration in spray ink could serve as effective "building blocks" for stacking and reforming as desired micro-nano structure. The transmission electron microscopy (TEM) morphologies of the coatings prepared by spraying the inks containing the so-called "building blocks" are demonstrated in Figure 1 (a) to (e). Their morphologies vary from mono-dispersed nano spheres to spongia-like agglomerates. The sample P0 demonstrates spherical particles with the diameter of about $40-50 \mathrm{~nm}$. These spherical particles were well dispersed in ethanol with no significant inter-particle connections. Adding CTAB surfactant into the solution at the beginning of the reaction between tetraethylorthosilicate (TEOS) and ammonium solution will result in the hydrophilic heads of CTAB attaching the formed $\mathrm{SiO}_{2}$ nucleus and the hydrophobic tails connecting each other and therefore the agglomerates generate. The more CTAB in the system, the larger size the agglomerates would be. When $0.05 \mathrm{~g}$ CTAB was added into the solution, the diameter of the particles (P1) in the coating was reduced to $10-20 \mathrm{~nm}$ (Figure $1(b)$ ), which could be attributed to the surfactant CTAB coated 
on the $\mathrm{SiO}_{2}$ nucleus via the interaction between the hydroxy groups of $\mathrm{SiO}_{2}$ and the hydrophilic ammonium head of the CTAB, refraining the $\mathrm{SiO}_{2}$ particles from further growing up. Samples P1-P3 present many nanoparticles $(10-20 \mathrm{~nm})$ agglomerating together into a spongia-like structure, and the size of these particle agglomerates in the coatings increases (see the red dotted circles) with the CTAB content in the spray inks and they are about 100-500 nm (Figure 1 (b), P1), 0.5-1.5 $\mu \mathrm{m}$ (Figure 1 (c), P2), 2-4 $\mu \mathrm{m}$ (Figure 1 (d), P3), respectively. Further increasing CTAB the silica particles become even larger blocks with the size of the agglomerates approaching $5 \mu \mathrm{m}$ (Figure 1 (e), P4). Figure 1 (f) shows the schematic diagram of the prepared superhydrophobic and superamiphiphobic surfaces. When spraying the inks with the different degrees of agglomeration, the nanostructures of the films are quite different. The nanoparticles are agglomerated together becoming micro particles, and when these agglomerated particles are sprayed on the substrate, the micro-nano over-hang structures are constructed. For the superamphiphobic films, the formation of micro-nano over-hang structure is a key point.

The SCAs of liquids having the different surface tensions on the as-made films are shown in Figure 2 (a). These liquids include water, glycol, oleic acid, hexadecane and dodecane with the surface tensions of $72 \mathrm{mN} / \mathrm{m}, 48.4 \mathrm{mN} / \mathrm{m}, 33.8 \mathrm{mN} / \mathrm{m}, 27.3 \mathrm{mN} / \mathrm{m}$ and $25.3 \mathrm{mN} / \mathrm{m}$ respectively. It was found that the SCAs of the liquids decrease with the decrease of surface tension $(\gamma)$ from water to dodecane. The SCAs of these liquids on the glass (G) are all less than $90^{\circ}$, but on the PFTS-coated glasses (PFTS=1H, 1H, 2H, 2H-perfluorodecyltrichlorosilane), the SCAs of these liquids increase obviously, with the highest value of $114^{\circ}$ for water (Sample F/G in Fig 2 (a)). As shown in the Figure 2 (a) (yellow dotted box), the F/P0 film only has the super-repellence for water, Aappwater $=163 \pm 3^{\circ} ; \mathrm{F} / \mathrm{P} 1$ film is super-repellent both to water and glycol, $\theta$ appwater $=164 \pm 3^{\circ}, \theta^{\mathrm{app}}$ glycol $=152 \pm 3^{\circ}$; F/P2 film has the good superamphiphobic property for water, glycol and oleic 
acid, appwater $=165 \pm 3^{\circ}$, ${ }_{\text {appglycol }} 162 \pm 3^{\circ}, \theta^{\text {app }}$ oleic acid $=157 \pm 3^{\circ}$; It needs to be noted that F/P3 film shows excellent superamphiphobic property for all liquids (see the red dotted box), $\theta^{\text {app }}{ }_{\text {water }}=166 \pm 3^{\circ}$,

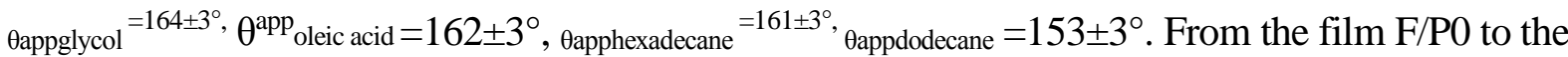
film F/P3, the SCAs of the same liquids increase. The improved superamphiphobic properties are probably due to the generation of micro-nano over-hang topographies of films prepared by using different silica building blocks.

Figure 2 (b)-(f) shows the SEM images of the films made by spray-coating the particles P0-P4, respectively. The insets in Figure 2 (b)-(f) show the SEM images of the corresponding films with higher amplification. The morphology of the films becomes rougher from the films F/P0 to F/P3. It is observed that Film F/P0 has the cracks (Figure 2 (b)), which is due to the violent vaporization of ethanol after the film was sprayed on the substrate. The temperature of the substrate during coating could generate thermal stress in the films and thus influence the topography of the films (Figure S1-S2). The best substrate temperature is found to be $200{ }^{\circ} \mathrm{C}$. The SEM image of the film F/P0 (Figure 2 (b) inset) shows that the film is composed of many nano spherical particles that are closely packed. The film F/P1 (Figure 2 (c)) possesses a similar morphology to F/P0 at low magnification and also shows the cracks. However, the observation by high magnification SEM reveals that a refined nanostructure with pore channels starts to form. It is observed that the film F/P2 (Figure 2 (d)) has no cracks, which is because the holes generated in the $\mathrm{SiO}_{2}$ agglomeration are larger than those holes observed in the films $\mathrm{F} / \mathrm{P} 0$ and $\mathrm{F} / \mathrm{P} 1$, and the vapor could escape from the holes and thus the thermal stress is released in the porous film. The pores in the film F/P3 and over-hang structure (dotted red cycles in Figure 2 (e)) can be obviously observed in Figure 2 (e). It is also observed that the over-hang structure is composed of many small $\mathrm{SiO}_{2}$ nanoparticles in $\mathrm{F} / \mathrm{P} 3$ that were not observed in the samples $\mathrm{F} / \mathrm{P} 0$, 
$\mathrm{F} / \mathrm{P} 1$ and F/P2. The formation of over-hang structure is due to, firstly, the micro-size agglomerates, and secondly, the removal of CTAB. Although micro-size holes were formed in the film F/P4 (Figure 2 (f)), the large blocks of agglomerated nanoparticles could not completely cover the substrate and nano particles structure is too large so that the low surface tension liquids can easily penetrate to the bottom of the film, leading to the fact that the film F/P4 can only super-repel water. It is conspicuous that the morphologies of the 5 films are different from each other, which can be attributed to the variation of CTAB content and the difference of resultant microstructures in the silica building blocks. As shown in Figure 1, the degree of the particle agglomeration is enhanced when more CTAB are added. The bigger chunks of particles in the $\mathrm{SiO} 2$ building-blocks are the easier to form micron-sized porous films. At the same time, the removal of CTAB that entangled with silica can also produce nano holes in the films. The best superamphiphobic film F/P3 should be ascribed to the addition of proper amount of $\mathrm{CTAB}$, which results in the proper degree of agglomeration of $\mathrm{SiO}_{2}$ nanoparticles. The SEM cross section image (Figure S3) of the films F/P0 - F/P3 and stylus profiler curves (Figure S4) obviously show more micro-nano over-hang structures of the surfaces in $\mathrm{F} / \mathrm{P} 3$, which allows more air trapped in this film, resulting in a smaller value of the $f$ in Equation (1). In the case of the same liquid, the smaller value of the $f$ will lead to larger $\theta^{\text {app }}$ when the rf remains no change in Equation (1).

\section{Highly Transparent Superamphiphobic Films}

High transparency of the superamphiphobic films enables them to find applications in visual windows in buildings, cars, displays and etc. However the designed micro-nano over-hang structure that brings film roughness normally causes intensive light scattering, which greatly reduces the transparency. Although the thicknesses of the films P0-P4 are all about $5 \mu \mathrm{m}$ (Figure 
S3), the transmittance of the films is widely various (Figure S5 and S6). According to Uv-Vis measurements (Figure S6), the transmittance of the films P0, P1, P2, P3 and P4 are about 89.3\%, $90.4 \%, 80.4 \%, 73.6 \%$, and 81\%, respectively. Film P3 has the lowest transmittance, which can be attributed to its special micro-nano over-hang structure, in which small particles agglomerate forming micron-size particles and many holes inside resulting in strong light scattering, though it shows the best superamphiphobic performance among all the films.

To improve the transmittance of the film P3, the thickness of the film P3 was reduced by using the diluted spraying ink. Figure 3 (a) shows the images of the as-made superamphiphobic films that have different thicknesses and have water (containing methyl blue), olive oil (yellow), hexadecane, dodecane drops on top of them. The Uv-vis characterization (Figure 3 (b)) suggests that the average transmittance between wavelength $350 \mathrm{~nm}$ to $800 \mathrm{~nm}$ is $89 \%$ for P3-C1 (395 nm thick), which is very close to the glass substrate, 90.3\%. Only P3-C4 (3559 nm thick) can superrepel the low surface tension liquid, dodecane, with transparency up to $73.6 \%$. The detailed date of SCAs results are shown in Figure 3 (c). It can be noticed that the highly transparent samples can still be superamphiphobic for water (blue) and olive oil (yellow) with very low haze (Figure $3(\mathrm{c})$ ).

As shown in Figure 3 (d), the average film thickness is $395 \mathrm{~nm}, 1595 \mathrm{~nm}, 2491 \mathrm{~nm}$, and $3559 \mathrm{~nm}$ for P3-C1, P3-C2, P3-C3 and P3-C4, respectively. The film thickness increases with $\mathrm{SiO}_{2}$ content in the spraying ink because in every single fog drop in spraying liquid contains more $\mathrm{SiO}_{2}$ particles. Using the inks with higher $\mathrm{SiO}_{2}$ particle content would also result in more $\mathrm{SiO}_{2}$ particles compiling in the same area leading to larger roughness. Figure 3 (d) shows that the average height of the peaks is $\sim 486 \mathrm{~nm}$ for film $\mathrm{P} 3-\mathrm{C} 1, \sim 2,072 \mathrm{~nm}$ for film $\mathrm{P} 3-\mathrm{C} 2, \sim 4,473 \mathrm{~nm}$ for film $\mathrm{P} 3-\mathrm{C} 3, \sim 6,300 \mathrm{~nm}$ for film $\mathrm{P} 3-\mathrm{C} 4$, respectively. The average interval of the peaks is 
21.3 Jim, 23.4 Jim, 21.4 Jim, 20.62 Jim for the films P3-C1, P3-C2, P3-C3, P3-C4, respectively. There would have more air trapped in the films that have higher peaks and more over-hang structures. The liquids are harder to penetrate the thicker films and reach to the bottom if the film possesses stronger peaks in over-hang structure, which also results in larger light scattering and less transparency.

\section{Robust and Self-healing Superamphiphobic Films}

In outdoor environment, superamphiphobic surfaces need to survive harsh conditions, like sand abrasion by wind and the adsorption of small organic molecules, which can change the roughness and the surface tension of the film leading to the loss of efficacy. In order to improve the mechanical properties of the films, titanium diisopropoxide bis 2, 4-pentanedionate (TAA) was added to the $\mathrm{SiO}_{2}$ system to act as a binder of $\mathrm{SiO}_{2}$ particles after it was pyrolysed. The films obtained with the addition of different TAA are referred as P3-T0, P3-T1, P3-T2, P3-T3 and P3T4 (see Experimental for details). To investigate the mechanical resistance of the films with the addition of TAA, sand abrasion test (Figure 4 (a)-(c)) and pencil test (Figure 4 (d)) were performed. Sand grains $(200 \mathrm{~g}, 10 \mathrm{~g} / \mathrm{s})$ with a diameter of 0.6 to $0.8 \mathrm{~mm}$ impinged the P3-T3 surface from a height of $24 \mathrm{~cm}$. Figure 4 (b) and (c) are the microscope images of the films P3-T0 (without TAA) and P3-T3 after sand abrasion. The film P3-T0 after the abrasion test was partially peeled off (the red dotted circles in Figure 4 (b)), indicating that the P3-T0 silica surfaces were not sufficiently robust to withstand the impact of sand abrasion. The mechanical property of the film P3-T3 was greatly improved, with only a small impact on the film being observed (Figure 4 (c), the red dotted circles). However, although the films partially destroyed by the sand abrasion, the

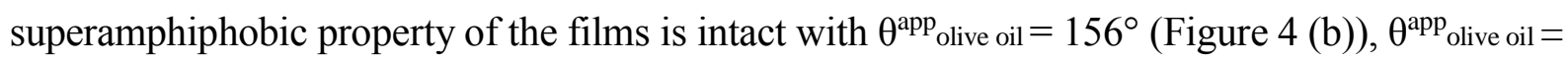
$154^{\circ}$ (Figure 4 (c)). Figure 4 (d) shows the curves of stylus profiler of 
the pencil scratch on the films P3-T0, P3-T1, P3-T2, P3-T3, and P3-T4, respectively. The pencil scratches left obvious marks on the films P3-T0, P3-T1, P3-T2. The marks by pencil scratch on the films P3-T3 and P3-T4 could not be obviously observed while film P3-T0 was totally scraped off (see the Figure S7 and S8), indicating that the mechanical strength of the films was improved upon the addition of the appropriate amount of $\mathrm{TiO}_{2}$ binder.

Furthermore, the inclusion of $\mathrm{TiO}_{2}$ adds photocatalytic ability which enables recoverability of the superamphiphobic films degraded by organic contamination. Figure 4 (e) shows the Uv-vis curves of the film P3-T3 with and without methylene blue ( $\mathrm{Mb}$ and $0 \mathrm{Mb})$ after photocatalytic decomposition of $\mathrm{Mb}$ for different illumination time (0-90 min). The spectral absorption (500600 $\mathrm{nm}$ ) of $\mathrm{Mb}$ was weakened when the time of the light exposure prolongs. Figure 4 (f) is the image and SCAs of the water and olive oil on the different P3-T3 films (0 Mb, 0 min, $90 \mathrm{~min})$. The SCAs of the water and olive oil on the P3-T3 without $\mathrm{Mb}(0 \mathrm{Mb})$ is $163^{\circ}$ and $159^{\circ}$, respectively. However when the P3-T3 film coated with Mb, the liquid-repellent property decreases and therefore the superamiphiphobic ability losses, $\theta_{a p p w a t e r}=102^{\circ}, \theta^{\text {app }}$ olive oil $=66^{\circ}$, which is attributed to the contamination of the surface. The photocatalytic $\mathrm{TiO}_{2}$ can decompose the small hydrocarbon materials, and after the illumination for $90 \mathrm{~min}$, the $\mathrm{Mb}$ almost totally disappears. The most important phenomenon is that the superamphiphobic property was fully recovered, Aappwater $^{2}=160^{\circ}$, $\theta^{\mathrm{app}}$ olive oil $=154^{\circ}$. This excellent self-healing property is very practical for outdoor environment where micro/nano organic pollutants are adsorbed on the surface.

Figure 5 (a) is the SEM image of the film P3-T3. It shows the basic over-hang structure still existing on the surface of the film after the addition of TAA. Its low and high resolution TEM images show the edge of the big particles (red dotted blocks) in Figure 5 (b). The calculation from the distinct lattice fringes shows that the distance between the two crystal planes is about 
$0.350 \mathrm{~nm}$, which is the (101) face of anatase. There are many similar small black particles around the big particles, which are assigned to anatase (yellow dotted circles) in the TEM images. The big particles are surrounded by the nano- $\mathrm{TiO}_{2}$ particles, and the diameter of the nano-anatase is about $10-20 \mathrm{~nm}$ (yellow dotted circles). Such configuration is effective to improve the mechanical property of the superamphiphobic film. The superamphiphobic properties will be decreased if too much TAA are added because the holes previously existed in the film (Figure 5(a)) will be filled with the small anatase particles, which decreases the volume of the over-hang structure. The P3-T3 film is found to be able to super-repel both water and oils, but the film P3T4 with more addition of TAA can super-repel only water (Figure S9). Hence, appropriate amount of the bolting titania is crucial to obtain robust and superamphiphobic films.

Figure 5 (c) shows the schematic diagram of how the mechanical properties of superamphiphobic films are strengthen and how photocatalytic function performs by the addition of anatase. The superamphiphobic films are sintered at $550^{\circ} \mathrm{C}$, at which temperature $\mathrm{SiO}_{2}$ cannot be sintered, however, this temperature is high enough to crystallize $\mathrm{TiO}_{2}$ so that it can help bolt $\mathrm{SiO}_{2}$ particles together as shown in Figure 5 (c), which leads to the increase of the mechanical property of the films. $\mathrm{TiO}_{2}$ can also act as a photocatalyst to assist the decomposition of organic contaminants attached on the superamphiphobic surfaces. When the $\mathrm{TiO}_{2}$ absorbs UV light, electron-hole pairs will be generated, which will oxidize the organic substances on the superamphiphobic surfaces, resulting in the decomposition of the organic contaminant. 52 The superhydrophobic windows have the potential applications as self-cleaning surfaces. Film P1 and a plain glass slide were both placed in outdoor environment for 30 days. Figure S10 shows the results of the self-cleaning tests. It has shown that after test in smoggy weather in Wuhan, the glass was covered with dirt while the P1 film is still clean and remains 


\section{CONCLUSIONS}

In summary, we present a facile route by spraying the inks containing the micro-sized silica agglomerates, a designed-structure building block, on glasses to form superamphiphobic films that possess robust, transparent and self-healing properties. The morphology, the degree of agglomeration, organic/inorganic ratio and particle size of the prepared silica building-blocks are adjusted by the amount of CTAB added in the precursor solution, which are crucial for obtaining low f factor and thus large SCAs for liquids. Optimized films demonstrated superamphiphobic properties. The best film could super-repel the oil with the surface tension as low as $25.3 \mathrm{mN} / \mathrm{m}$, and its SCA for water as high as $166^{\circ}$. For a film with the average thickness $395 \mathrm{~nm}$, its transmittance can be as high as $89 \%$ close to that of glass substrates $(90.3 \%)$.

Furthermore, the introduction of inorganic binder of crystallized anatase can help cement silica building blocks, which largely improves the mechanical property of the film (withstanding $2 \mathrm{H}$ pencil test). Moreover this unique nano structure also provides a photocatalytic property and can decompose organic contaminants upon exposing to lighting.

\section{EXPERIMENTAL SECTION}




\section{Materials}

Tetraethylorthosilicate (TEOS), cetyltrimethylammonium bromide (CTAB, ? 99\%), ethanol (? 99.7\%), olive oil, oleic oil, ammonia solution (25\%-28\%), hexane (? 97\%), glycol, methylene blue were obtained from Sino Pharm Chemical Reagent Co. Ltd. Dodecane (>99\%), hexadecane (98\%), 1H,1H,2H,2H-perfluorodecyltrichlorosilane(PFTS) were purchased from Aladdin. Titanium diisopropoxide bis 2, 4-pentanedionate (TAA, 75wt\% in isopropyl alcohol) was obtained from Alfa Aesar. All of the mentioned reagents were used as received.

\section{Experimental procedure}

Preparation of Different Agglomerated Silica Nanoparticles: a certain amount of CTAB was dissolved in a mixed solution composed of $4 \mathrm{~mL}$ ammonia solution and $80 \mathrm{~mL}$ ethanol. The mixture was stirred with a magnetic stirrer for 5 min till the CTAB was fully dissolved. $2 \mathrm{ml}$ of TEOS was dissolved in $80 \mathrm{ml}$ ethanol. This TEOS solution was quickly dripped into the CTAB solution. The resulting mixture was stirred at $40{ }^{\circ} \mathrm{C}$ for $12 \mathrm{~h}$. The obtained solid-liquid mixture was dried in air at $80{ }^{\circ} \mathrm{C}$ for $12 \mathrm{~h} . \mathrm{SiO}_{2}$ particle agglomerates with 5 different sizes were obtained by using the different amounts of the CTAB $(0 \mathrm{~g}, 0.05 \mathrm{~g}, 0.15 \mathrm{~g}, 0.25 \mathrm{~g}$ and $0.35 \mathrm{~g})$ and denoted as $\mathrm{P} 0, \mathrm{P} 1, \mathrm{P} 2, \mathrm{P} 3$ and $\mathrm{P} 4$, respectively.

Preparation of Different Superamphiphobic Films: The obtained (P0, P1, P2, P3 and P4) $\mathrm{SiO}_{2}$ particle agglomerates $(0.3 \mathrm{~g})$ were ultrasonically dispersed in $35 \mathrm{ml}$ ethanol solution, respectively. Soda-lime-silica glasses were used as substrates that were washed with ultrapure water and ethanol. The cleaned substrates were heated to about $200{ }^{\circ} \mathrm{C}$, and then $4 \mathrm{ml}$ of ultrasonically-dispersed $\mathrm{SiO}_{2}$ particles ink were sprayed onto an area of $2.5 \times 2.5 \mathrm{~cm}^{2}$ substrate at a distance of 
$15 \mathrm{~cm}$ at a rate of $4 \mathrm{ml} / \mathrm{min}$. The coated glass substrates were sintered in air at $550{ }^{\circ} \mathrm{C}$ for $1 \mathrm{~h}$ and cooled naturally. The coated substrates after sintered were dipped into hexane solution containing $1 \mathrm{wt} \%$ PFTS for $1 \mathrm{~min}$, dried naturally in air for $30 \mathrm{~min}$ and then the coated substrates were put onto a heating platform at $420^{\circ} \mathrm{C}$ for 3 min to volatilize redundant PFTS.

Prepare Different Transparent Superamphiphobic Films: A certain amount of P3 particles (0.05 g, $1.8 \mathrm{wt} \% ; 0.1 \mathrm{~g}, 3.6 \mathrm{wt} \% ; 0.2 \mathrm{~g}, 7.2 \mathrm{wt} \%$ and $0.3 \mathrm{~g}, 10.8 \mathrm{wt} \%$ ) were ultrasonically dispersed in $35 \mathrm{ml}$ ethanol, and the spraying and fluorinating processes are the same as mentioned above. The obtained films were denoted as P3-C1, P3-C2, P3-C3, P3-C4, respectively. Furthermore, in order to enhance the robust of the films, P3 particles $(0.3 \mathrm{~g})$ were ultrasonically dispersed in 35 $\mathrm{ml}$ ethanol, and then a certain amount of TAA $(0,200 \mu 1,400 \mu 1,600 \mu 1,800 \mu 1)$ were added in $4 \mathrm{ml} \mathrm{P} 3$ particles in ethanol before spraying. The obtained films were denoted as P3, P3-T1, P3T2, P3-T3, P3-T4, respectively.

Characterization of the Coatings: The morphologies of the particles and the films were examined with transmission electron microscope (TEM, JEM-2100F STEM/EDS), scanning electron microscope (SEM, ULTRA PLUS-43-13) and optical microscope (Olympus BX51). The contact angle of various liquids on the films was measured by Contact Angle tester (Theta Lite, Biolin Scientific) in the sessile mode at room temperature. The model of the stylus profiler is Dektakxt. The transmittance of the films was measured by the ultraviolet and visible spectrophotometer (Uv-Vis, PerkinElmer). The pencil scratching test was carried out using a Pencil Hardness Tester according to the State Standard Testing Method (GB/T6739-1996, equivalent of American Society for Testing and Materials (ASTM) D3363). The pencil was held firmly against the film at a $45^{\circ}$ angle and pushed forward by the tester at a speed of $0.5 \mathrm{~mm} / \mathrm{s}$. 
The light source for photocatalytic degradation is from the Class AAA Solar Simulators (94023A, Newport).

\section{FIGURES CAPTIONS}

Figure 1. (a), (b), (c), (d), and (e) are the TEM images of the as-obtained particles P0, P1, P2, P3 and P4 on carbon nets, respectively. P0 contains spherical particles (40-50 nm), P1-P3 contain the spongia-like agglomerates of $\mathrm{SiO}_{2}$ nanoparticles $(10-20 \mathrm{~nm})$. P4 contains larger agglomerates (red dotted circles) of $\mathrm{SiO} 2$ nanopaticles $(200 \mathrm{~nm})$; (f) the schematic diagram of prepared superhydrophobic and superamiphiphobic surfaces. The different sized nanoparticles create different sized agglomerates, which wereobtained by adjusting the content of CTAB. The resultant films are either superhydrophobic or superamphiphobic.

Figure 2. (a) the SCAs of different liquids: Water, Glycol, Oleic acid, Hexadecane, Dodecane on glass (G), PFTS coated glass (F/G), PFTS/P0 coated glass (F/P0), PFTS/P1 coated glass (F/P1), PFTS/P2 coated glass (F/P2), PFTS/P3 coated glass (F/P3), PFTS/P4 coated glass (F/P4); (b), (c), (d), (e), and (f) the low and high amplification (the insets on top right corner) SEM images of the as-obtained films F/P0, F/P1, F/P2, F/P3, F/P4, respectively; (g) the picture of the liquid droplets on the F/P3 film (water was added methyl blue to improve the comparison).

Figure 3. Optical measurements of the films: P3-C1, P3-C2, P3-C3 and P3-C4, (a) Optical pictures of the liquids, water (added methyl blue) and olive oil (yellow), hexadecane, dodecane on the superamphiphobic glasses ; (b) Transmittance of the surfaces; (c) SCAs of the different 
liquids on the surfaces; (d) stylus profiler of the surfaces (the inset of the Figure 3 (d) is the expansion of the vertical coordinate when horizontal coordinate remains unchanged).

Figure 4. (a) Optical picture of sea sand used for the sand abrasion tests (the average grain size is between 0.6-0.8 mm ), and the inset is the schematic diagram of sand abrasion tests; (b) and (c) Optical microscope images of the films P3-T0 and P3-T3 showing sand abrasion areas (red dotted cycles) and without and the insets are the SCAs of the olive oil drops deposited on the films after sand impingement); (d) stylus profiler of the pencil scratch on the films P3-T0, P3-T1, P3-T2, P3T3, and P3-T4, respectively; (e) the Uv-Vis transmittance of Film P3-T3 with methylene blue (Mb) photocatalytically degarded at different illumination time (0, 30, 60, and $90 \mathrm{~min})$; (f) the optical images and SCAs of the water and olive oil on the FilmP3-T3 (0 Mb presents no methylene blue; 0 min is the film with $\mathrm{Mb}$ under no illumination; $90 \mathrm{~min}$ is the film with $\mathrm{Mb}$ under a 90 min illumination).

Figure 5. (a) SEM image of the P3-T3; (b) TEM image of $\mathrm{SiO}_{2}$ particles bonded by $\mathrm{TiO}_{2}$; high resolution TEM image of Film P3-T3 (the yellow dotted circles are the anatase); (c) schematic diagram of $\mathrm{SiO}_{2}$ strengthen by anatase and photo degradation mechanism, $(\mathrm{CB}$, conduction band; VB, valence band).

\section{Displayed equations}

\section{ASSOCIATED CONTENT}

$$
\cos \theta={ }_{f} \cos \theta-1+
$$




\section{Supporting Information.}

The following files are available free of charge.

brief description (file type, i.e., PDF)

\section{AUTHOR INFORMATION}

\section{Corresponding Author}

*Q. Zhang. School of Aerospace, Transport and Manufacturing, Cranfield University, Cranfield, Bedfordshire, MK43 0AL, United Kingdom.q.zhang@ cranfield.ac.uk.

* J. Zhong. State Key Laboratory of Advanced Technology for Materials Synthesis and Processing, School of Materials Science and Engineering, Wuhan University of Technology, Wuhan 430070, China. jie.zhong@whut.edu.cn.

\section{Present Addresses}

$\dagger$ If an author's address is different than the one given in the affiliation line, this information may be included here.

\section{Author Contributions}

The manuscript was written through contributions of all authors. All authors have given approval to the final version of the manuscript. $\ddagger$ These authors contributed equally.

\section{Funding Sources}

Any funds used to support the research of the manuscript should be placed here (per journal style).

1. National Natural Science Foundation of China. NSFC, 51402115. 
2. Hubei Provincial Natural Science Foundation of China. 2016CFB464.

3. Fundamental Research Funds for the Central Universities. WUT, 2016IVA089, 2016 III030.

\title{
Notes
}

Any additional relevant notes should be placed here.

\section{ACKNOWLEDGMENT}

This work is financially supported by National Natural Science Foundation of China (NSFC 51402115), Hubei Provincial Natural Science Foundation of China (2016CFB464) and the Fundamental Research Funds for the Central Universities (WUT: 2016IVA089, 2016III030). JZ would like to thank the support of "Chutian Scholar Program" of Hubei Province, China. QZ would like to thank the support of "Hubei one-hundred Plan". The Analytical and Testing Center of Wuhan University of Technology is also acknowledged for the characterization support.

\author{
ABBREVIATIONS \\ CTAB, cetyltrimethylammonium bromide; SCAs, static contact angles;CVD, chemical vapour \\ deposition; TEOS, tetraethyl orthosilicate; TAA, titanium diisopropoxidebis 2,4-pentanedionate; \\ $\mathrm{Mb}$, methylene blue; $\mathrm{CB}$, conduction band; VB, valence band; PFTS, $1 \mathrm{H}, 1 \mathrm{H}, 2 \mathrm{H}, 2 \mathrm{H}-$ \\ perfluorodecyltrichlorosilane.
}

\section{REFERENCES}

1. Barthlott, W.; Neinhuis, C. Purity of the Sacred Lotus, or Escape from Contamination in Biological Surfaces. Planta 1997, 202 (1), 1-8.

2. Gao, X. F.; Jiang, L. Water-Repellent Legs of Water Striders. Nature 2004, 432 (7013), 36-36.

3. Michael, N. Materials Science: Slippery When Wetted. Nature 2011, 477 (7365), 412-3. 
4. Zheng, Y.; Gao, X.; Jiang, L. Directional Adhesion of Superhydrophobic Butterfly Wings. Soft Matter 2007, 3 (2), 178-182.

5. Bird, J. C.; Dhiman, R.; Kwon, H.-M.; Varanasi, K. K. Reducing the Contact Time of a Bouncing Drop. Nature 2013, 503 (7476), 385-8.

6. Bixler, G. D.; Bhushan, B. Bio-Inspired Rice Leaf and Butterfly Wing Surface Structures Combining Shark Skin and Lotus Effects. Soft Matter 2012, 8 (44), 11271-11284.

7. Yuan, T.; Meng, J.; Hao, T.; Wang, Z.; Zhang, Y. A Scalable Method toward Superhydrophilic and Underwater Superoleophobic PVDF Membranes for Effective Oil/Water Emulsion Separation. Acs Applied Materials \& Interfaces 2015, 7 (27), 14896-14904.

8. Su, B.; Tian, Y.; Jiang, L. Bio-Inspired Interfaces with Super Wettability: From Materials to Chemistry. Journal of the American Chemical Society 2016, 138 (6), 1727-1748.

9. Liu, K.; Jiang, L. Bio-Inspired Self-Cleaning Surfaces. Annual Review of Materials Research 2012, 42, 231-263.

10. Chen, K.; Zhou, S.; Wu, L. Self-Healing Underwater Superoleophobic and AntiBiofouling Coatings Based on the Assembly of Hierarchical Microgel Spheres. Acs Nano 2016, 10 (1), 1386-1394.

11. Cheng, Y.; Lu, S.; Xu, W.; Wen, H.; Wang, J. Fabrication of Superhydrophobic Au-Zn Alloy Surface on a Zinc Substrate for Roll-down, Self-cleaning and Anti-corrosion Properties. Journal of Materials Chemistry A 2015, 3 (32), 16774-16784. 
12. Lv, J.; Song, Y.; Jiang, L.; Wang, J. Bio-Inspired Strategies for Anti-Icing. Acs Nano 2014, 8 (4), 3152-3169.

13. Paven, M.; Papadopoulos, P.; Schoettler, S.; Deng, X.; Mailaender, V.; Vollmer, D.; Butt, H.-J. Super Liquid-Repellent Gas Membranes for Carbon Dioxide Capture and Heart-Lung Machines. Nature Communications 2013, 4(9), 2512-2512.

14. Wen, L.; Tian, Y.; Jiang, L. Bioinspired Super-Wettability from Fundamental Research to Practical Applications. Angewandte Chemie International Edition 2015, 54 (11), 3387-3399.

15. Wang, B.; Liang, W. X.; Guo, Z. G.; Liu, W. M. Biomimetic Super-Lyophobic and Super-Lyophilic Materials Applied for Oil/Water Separation: a New Strategy beyond Nature. Chemical Society Reviews 2015, 44 (1), 336-361.

16. Young, T. An Essay on the Cohesion of Fluids. Royal Society of London Philosophical Transactions 1805, 95, 65-87.

17. Wenzel, R. N. Resistance of Solid Surfaces to Wetting by Water. Ind.Eng.Chem 1936, 28 (8), 988-994.

18. Cassie, A. B. D.; Baxter, S. Wettability of Porous Surfaces. Transactions of the Faraday Society 1944, 40 (0), 546-551.

19. Wang, S.; Jiang, L. Definition of Superhydrophobic States. Advanced Materials 2007, 19 (21), 3423-3424.

20. Tsujii, K.; Yamamoto, T.; Onda, T.; Shibuichi, S. Super Oil-Repellent Surfaces. Angewandte Chemie International Edition 1997, 36(9), 1011-1012. 
21. Gao, L.; McCarthy, T. J. How Wenzel and Cassie were Wrong. Langmuir 2007, 23

(7), 3762-3765.

22. Liu, T. L.; Kim, C.-J. C. J. Turning a Surface Super-Repellent Even to Completely Wetting Liquids. Science 2014, 346 (6213), 1096-1100.

23. Deng, X.; Mammen, L.; Butt, H. J.; Vollmer, D. Candle Soot as a Template for a Transparent Robust Superamphiphobic Coating. Science 2012, 335 (6064), 67-70.

24. Mazumder, P.; Jiang, Y.; Baker, D.; Carrilero, A.; Tulli, D.; Infante, D.; Hunt, A. T.; Pruneri, V. Superomniphobic, Transparent, and Antireflection Surfaces Based on Hierarchical Nanostructures. Nano Letters 2014, 14 (8), 4677-4681.

25. Li, F.; Du, M.; Zheng, Q. Dopamine/Silica Nanoparticle Assembled, Microscale Porous Structure for Versatile Superamphiphobic Coating. Acs Nano 2016, 10 (2), 2910-2921.

26. Xiong, L.; Kendrick, L. L.; Heusser, H.; Webb, J. C.; Sparks, B. J.; Goetz, J. T.; Guo, W.; Stafford, C. M.; Blanton, M. D.; Nazarenko, S.; Patton, D. L. Spray-Deposition and Photopolymerization of Organic-Inorganic Thiolene Resins for Fabrication of Superamphiphobic Surfaces. Acs Applied Materials \& Interfaces 2014, 6 (13), 10763-10774.

27. Zhu, X.; Zhang, Z.; Ren, G.; Men, X.; Ge, B.; Zhou, X. Designing Transparent Superamphiphobic Coatings Directed by Carbon Nanotubes. Journal of Colloid and Interface Science 2014, 421, 141-145. 
28. Lee, S. Y.; Rahmawan, Y.; Yang, S. Transparent and Superamphiphobic Surfaces from Mushroom-Like Micropillar Arrays. ACS Applied Materials \& Interfaces 2015, 7 (43), 2419724203.

29. Khedir, K. R.; Saifaldeen, Z. S.; Demirkan, T. M.; Al-Hilo, A. A.; Brozak, M. P.; Karabacak, T. Robust Superamphiphobic Nanoscale Copper Sheet Surfaces Produced by a Simple and Environmentally Friendly Technique. Advanced Engineering Materials 2015, 17 (7), 982-989.

30. Ragesh, P.; Nair, S. V.; Nair, A. S. An Attempt to Fabricate a Photocatalytic and Hydrophobic Self-Cleaning Coating via Electrospinning. Rsc Advances 2014, 4 (73), 3849838504.

31. Feng, X. J.; Zhai, J.; Jiang, L. The Fabrication and Switchable Superhydrophobicity of $\mathrm{TiO}_{2}$ Nanorod Films. Angewandte Chemie International Edition 2005, 44 (32), 5115-5118.

32. Chen, K.; Zhou, S.; Yang, S.; Wu, L. Fabrication of All-Water-Based SelfRepairing Superhydrophobic Coatings Based on UV-Responsive Microcapsules. Advanced Functional Materials 2015, 25 (7), 1035-1041.

33. Sun, Y.; Wang, L.; Gao, Y.; Guo, D. Preparation of Stable Superamphiphobic Surfaces on Ti-6Al-4V Substrates by One-Step Anodization. Applied Surface Science 2015, $324,825-830$.

34. Wang, S.; Song, Y.; Jiang, L. Microscale and Nanoscale Hierarchical Structured Mesh Films with Superhydrophobic and Superoleophilic Properties Induced by Long-Chain Fatty Acids. Nanotechnology 2007, 18 (1). 
35. Zhou, H.; Wang, H.; Niu, H.; Fang, J.; Zhao, Y.; Lin, T. Super-Strong, Chemically Stable, Superamphiphobic Fabrics from Particle-Free Polymer Coatings. Advanced Materials Interfaces 2015, 2 (6).

36. Grozea, C. M.; Rabnawaz, M.; Liu, G.; Zhang, G. Coating of Silica Particles by Fluorinated Diblock Copolymers and Use of the Resultant Silica for Superamphiphobic Surfaces. Polymer 2015, 64, 153-162.

37. Milionis, A.; Dang, K.; Prato, M.; Loth, E.; Bayer, I. S. Liquid Repellent Nanocomposites Obtained from One-Step Water-Based Spray. Journal of Materials Chemistry A 2015, 3 (24), $12880-12889$.

38. Lu, Y. Robust Self-Cleaning Surfaces that Function When Exposed to Either Air or Oil. Science 2015, 347.

39. Li, Y.; Zhu, X.; Zhou, X.; Ge, B.; Chen, S.; Wu, W. A Facile Way to Fabricate a Superamphiphobic Surface. Applied Physics a-Materials Science \& Processing 2014, 115 (3), $765-770$.

40. Jiang, W.; Grozea, C. M.; Shi, Z.; Liu, G. Fluorinated Raspberry-Like Polymer Particles for Superamphiphobic Coatings. Acs Applied Materials \& Interfaces 2014, 6 (4), 2629-2638.

41. Li, B. C.; Zhang, J. P.; Gao, Z. Q.; Wei, Q. Y. Semitransparent Superoleophobic Coatings with Low Sliding Angles for Hot Liquids Based on Silica Nanotubes. Journal of Materials Chemistry A 2016, 4 (3), 953-960. 
42. Zhou, H.; Wang, H.; Niu, H.; Gestos, A.; Lin, T. Robust, Self-Healing

Superamphiphobic Fabrics Prepared by Two-Step Coating of Fluoro-Containing Polymer,

Fluoroalkyl Silane, and Modified Silica Nanoparticles. Advanced Functional Materials 2013, 23

(13), 1664-1670.

43. Zhang, G.; Lin, S.; Wyman, I.; Zou, H.; Hu, J.; Liu, G.; Wang, J.; Li, F.; Liu, F.;

Hu, M. Robust Superamphiphobic Coatings Based on Silica Particles Bearing Bi-Functional Random Copolymers. Acs Applied Materials \& Interfaces 2013, 5 (24), 13466-13477.

44. Geng, Z;; He, J. An Effective Method to Significantly Enhance the Robustness and Adhesion-to-Substrate of High Transmittance Superamphiphobic Silica Thin Films. Journal of Materials Chemistry A 2014, 2 (39), 16601-16607.

45. Kamegawa, T.; Shimizu, Y.; Yamashita, H. Superhydrophobic Surfaces with Photocatalytic Self-Cleaning Properties by Nanocomposite Coating of $\mathrm{TiO}_{2}$ and Polytetrafluoroethylene. Advanced Materials 2012, 24 (27), 3697-3700.

46. Chen, K. L.; Wu, Y.; Zhou, S. X.; Wu, L. M. Recent Development of Durable and Self-Healing Surfaces with Special Wettability. Macromolecular Rapid Communications 2016, 37 (6), 463-485.

47. Zhou, S.; Ding, X.; Wu, L. Fabrication of Ambient-Curable Superhydrophobic Fluoropolysiloxane/ $\mathrm{TiO}_{2}$ Nanocomposite Coatings with Good Mechanical Properties and Durability. Progress in Organic Coatings 2013, 76 (4), 563-570.

48. Zhao, Y.; Liu, Y.; Xu, Q.; Barahman, M.; Lyons, A. M. Catalytic, Self-Cleaning Surface with Stable Superhydrophobic Properties: Printed Polydimethylsiloxane (PDMS) Arrays Embedded with $\mathrm{TiO}_{2}$ Nanoparticles. Acs Applied Materials \& Interfaces 2015, 7 (4), 2632-2640. 
49. Stöber, W.; Fink, A.; Bohn, E. Controlled Growth of Monodisperse Silica Spheres in the Micron Size Range. Journal of Colloid and Interface Science 1968, 26 (1), 62-69.

50. Aulin, C.; Yun, S. H.; Wagberg, L.; Lindstrom, T. Design of Highly Oleophobic Cellulose Surfaces from Structured Silicon Templates. Acs Applied Materials \& Interfaces 2009, 1 (11), 2443-2452.

51. Zhang, A.; Gu, L.; Hou, K.; Dai, C.; Song, C.; Guo, X. Mesostructure-Tunable and SizeControllable Hierarchical Porous Silica Nanospheres Synthesized by Aldehyde-Modified Stöber method. Rsc Advances 2015, 5 (72), 58355-58362.

52. Linsebigler, A. L.; Lu, G.; Yates, J. T. Photocatalysis on $\mathrm{TiO}_{2} \mathrm{Surfaces}_{\text {: Principles, }}$ Mechanisms, and Selected Results. Chem. Rev 1996, 95, 735-758. 

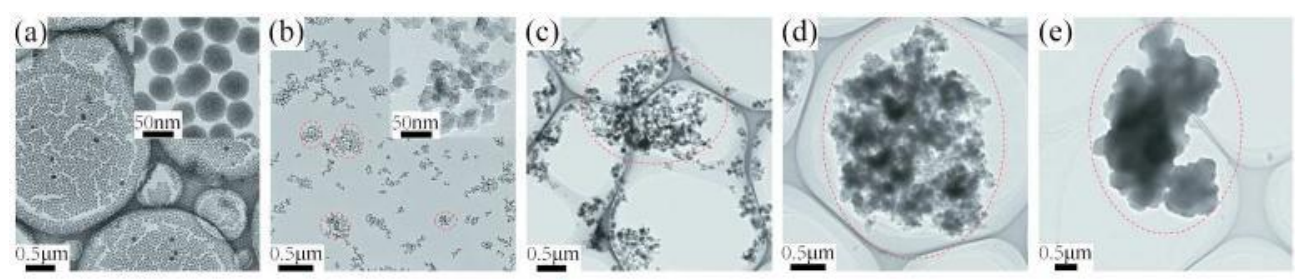

(f)
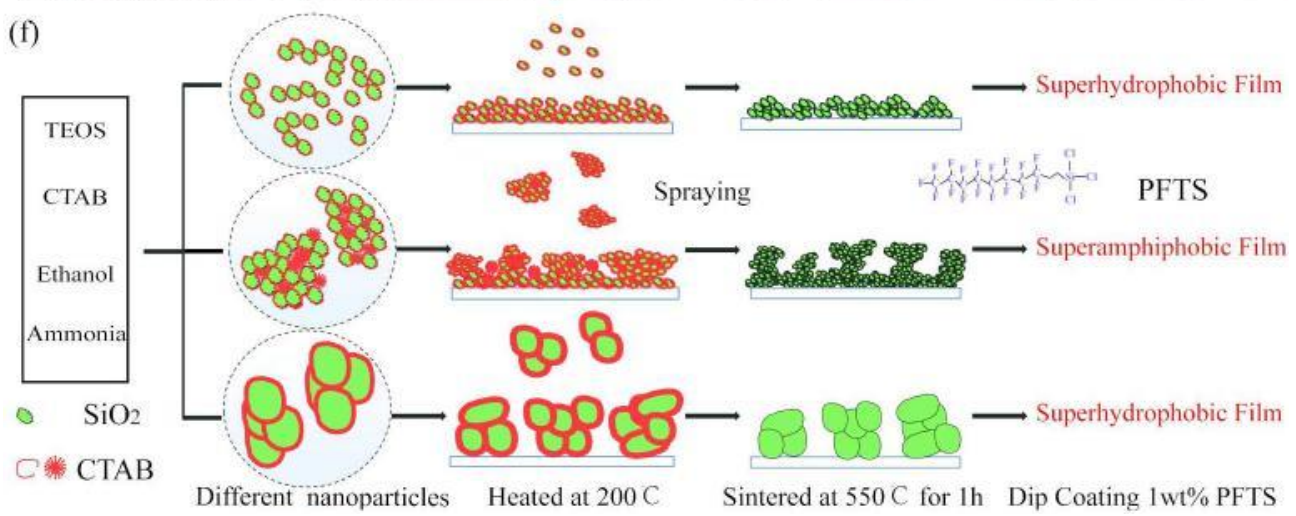

Figure 1. (a), (b), (c), (d), and (e) are the TEM images of the as-obtained particles P0, P1, P2, P3 and P4 on carbon nets, respectively. P0 contains spherical particles (40-50 nm), P1-P3 contain the spongia-like agglomerates of SiO2 nanoparticles (10-20 nm). P4 contains larger agglomerates (red dotted circles) of $\mathrm{SiO} 2$ nanopaticles $(200 \mathrm{~nm})$; (f) the schematic diagram of prepared superhydrophobic and superamiphiphobic surfaces. The different sized nanoparticles create different sized agglomerates, which wereobtained by adjusting the content of CTAB. The resultant films are either superhydrophobic or superamphiphobic.

$205 \times 125 \mathrm{~mm}(300 \times 300$ DPI $)$ 

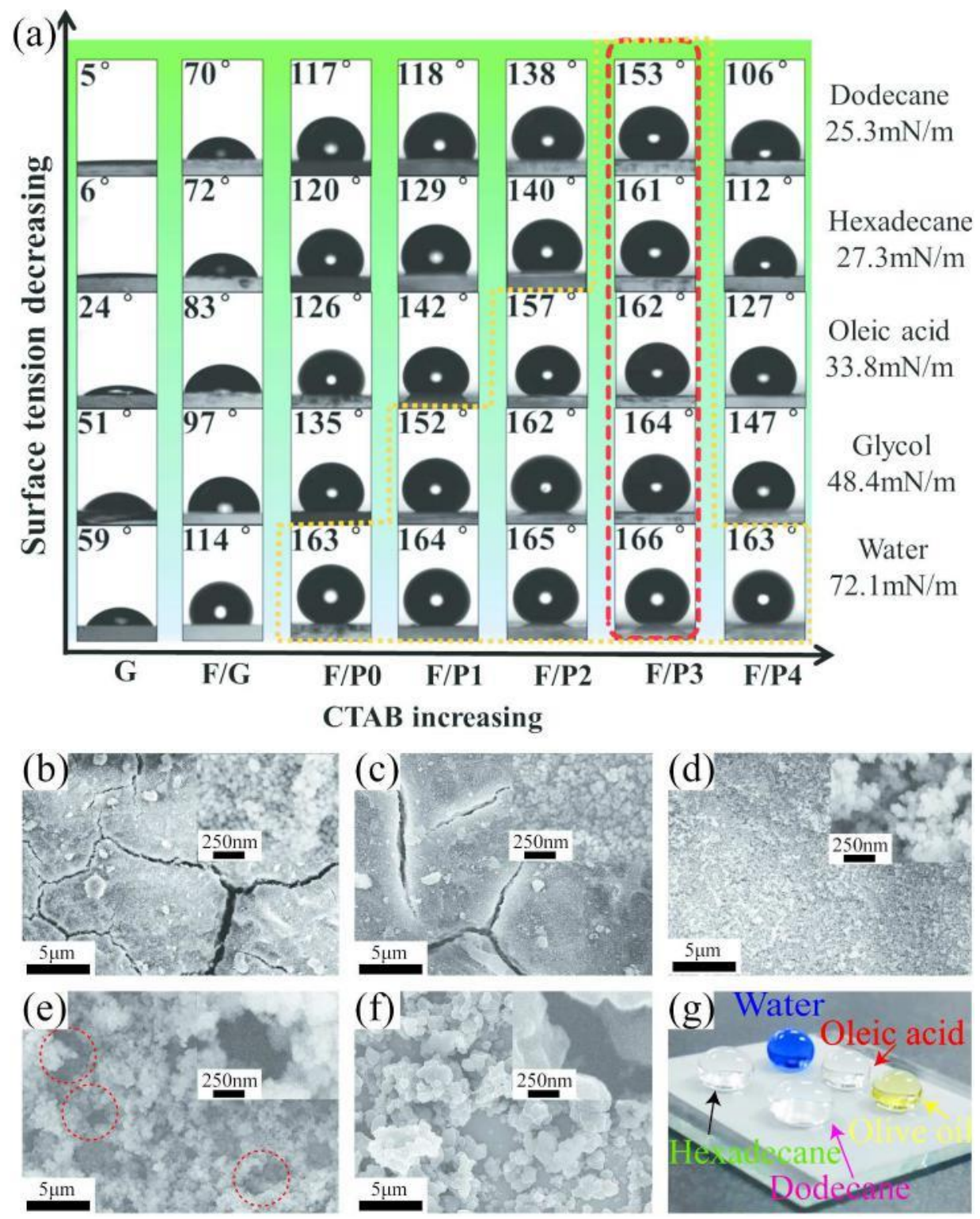

Figure 2. (a) the SCAs of different liquids: Water, Glycol, Oleic acid, Hexadecane, Dodecane on glass (G), PFTS coated glass (F/G), PFTS/P0 coated glass (F/P0), PFTS/P1 coated glass (F/P1), PFTS/P2 coated glass (F/P2), PFTS/P3 coated glass (F/P3), PFTS/P4 coated glass (F/P4); (b), (c), (d), (e), and (f) the low and high amplification (the insets on top right corner) SEM images of the as-obtained films F/P0, F/P1, F/P2, F/P3, F/P4, respectively; $(\mathrm{g})$ the picture of the liquid droplets on the F/P3 film (water was added methyl blue to improve the comparison). 

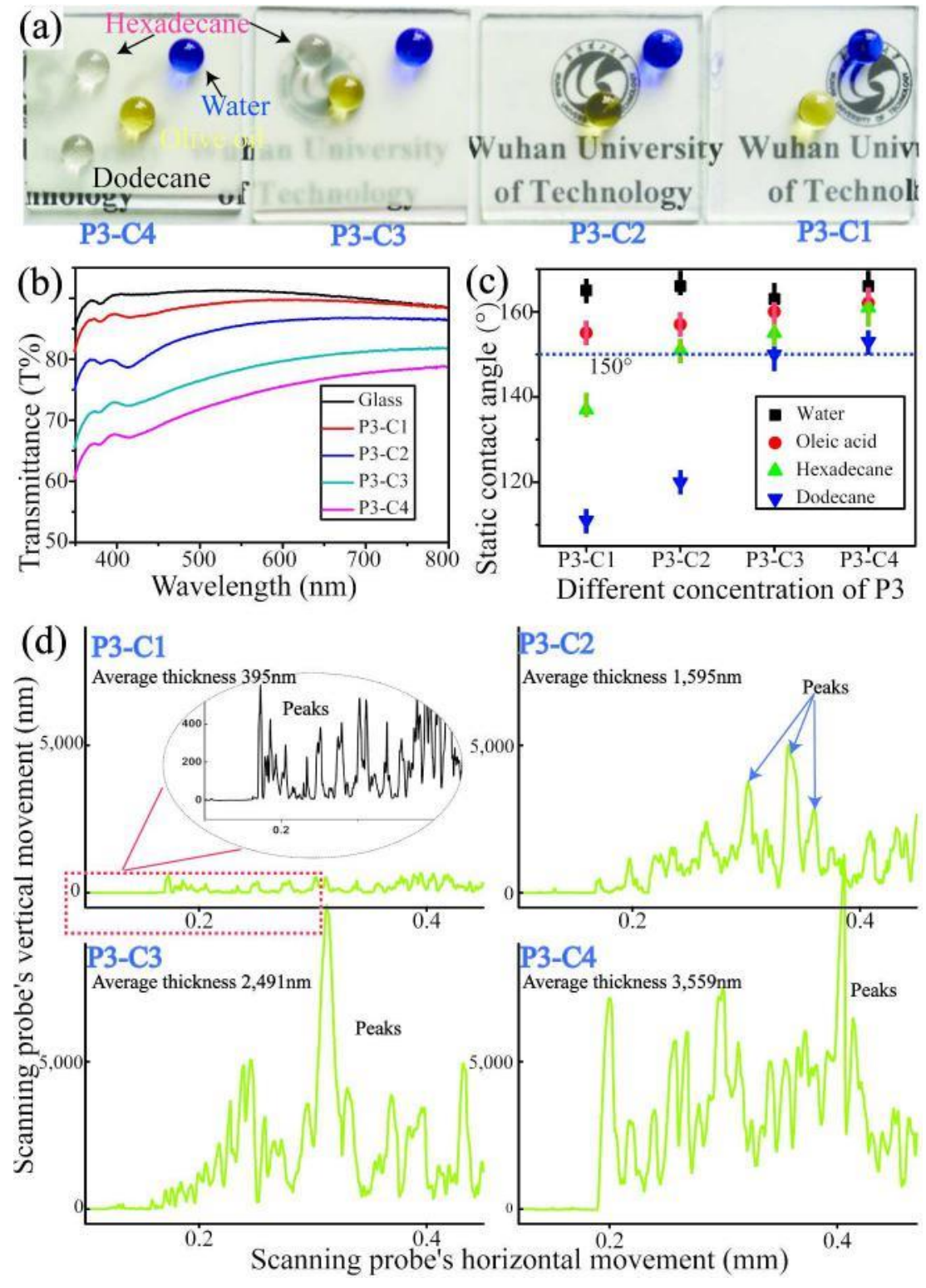

Figure 3. Optical measurements of the films: P3-C1, P3-C2, P3-C3 and P3-C4, (a) Optical pictures of the liquids, water (added methyl blue) and olive oil (yellow), hexadecane, dodecane on the superamphiphobic glasses ; (b) Transmittance of the surfaces; (c) SCAs of the different liquids on the surfaces; (d) stylus profiler of the surfaces (the inset of the Figure 3 (d) is the expansion of the vertical coordinate when horizontal coordinate remains unchanged). 

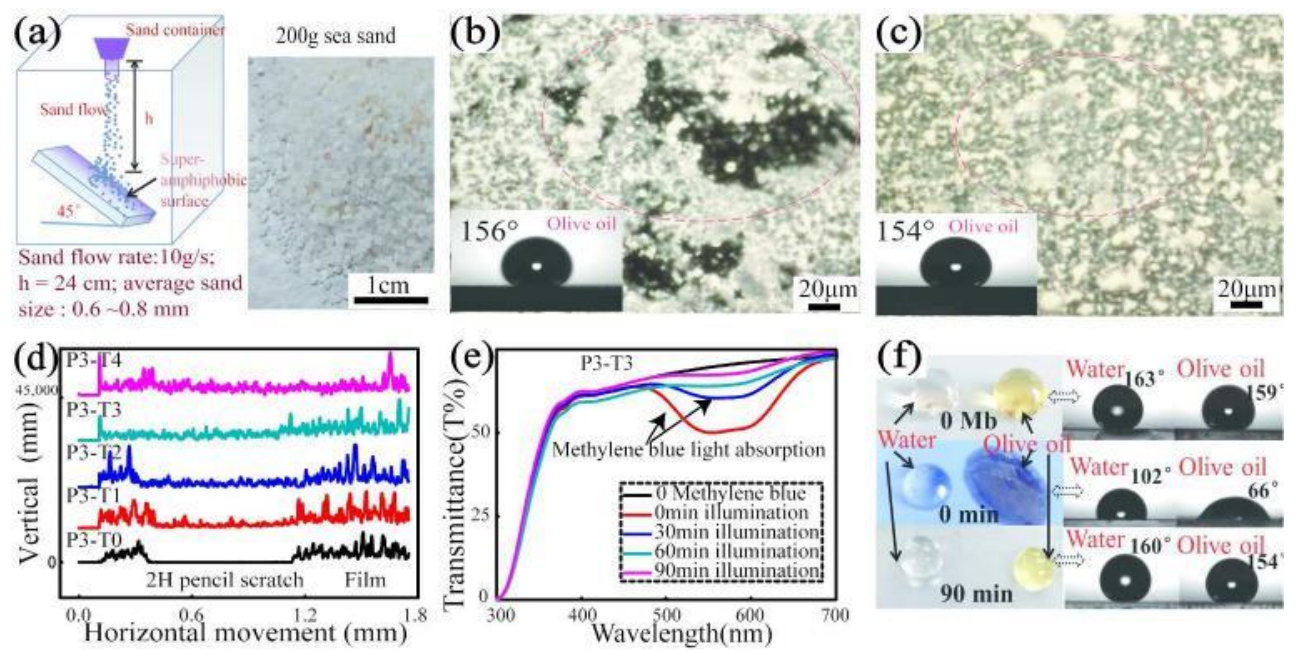

Figure 4. (a) Optical picture of sea sand used for the sand abrasion tests (the average grain size is between 0.6-0.8 $\mathrm{mm}$ ), and the inset is the schematic diagram of sand abrasion tests; (b) and (c) Optical microscope images of the films P3-T0 and P3-T3 showing sand abrasion areas (red dotted cycles) and without and the insets are the SCAs of the olive oil drops deposited on the films after sand impingement); (d) stylus profiler of the pencil scratch on the films P3-T0, P3-T1, P3-T2, P3-T3, and P3-T4, respectively; (e) the Uv-Vis transmittance of film P3-T3 with methylene blue (Mb) photocatalytically degarded at different illumination time (0, 30, 60, and $90 \mathrm{~min})$; (f) the optical images and SCAs of the water and olive oil on the film P3-T3 (0 $\mathrm{Mb}$ presents no methylene blue; $0 \mathrm{~min}$ is the film with $\mathrm{Mb}$ under no illumination; $90 \mathrm{~min}$ is the film with $\mathrm{Mb}$ under a 90 min illumination). 


\section{Page 31 of 31}

1 2

(a)
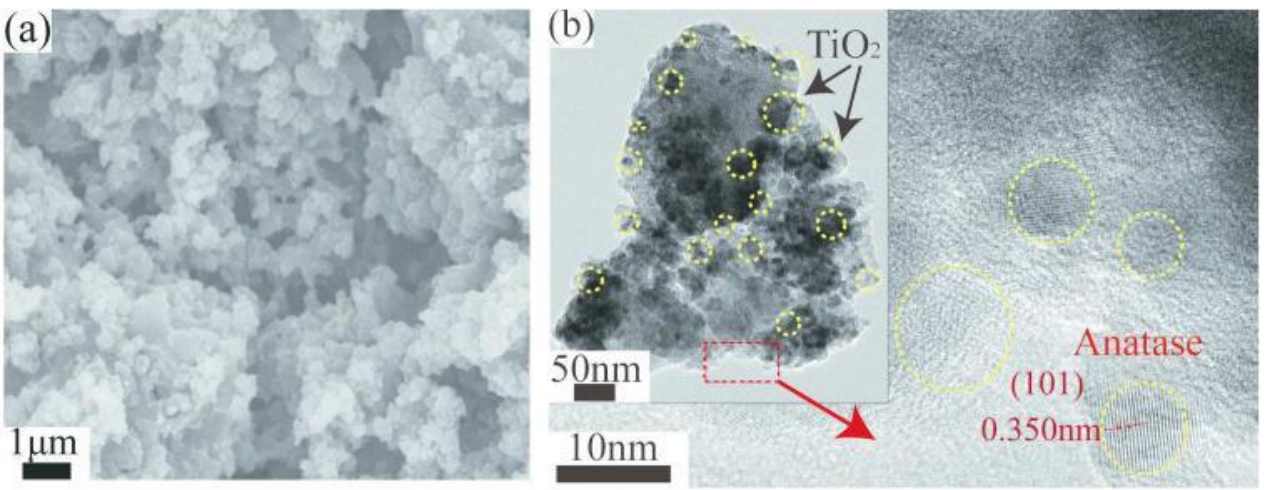

(c)

Figure 5. (a) SEM image of the P3-T3; (b) TEM image of SiO2 particles bonded by TiO2; high resolution TEM image of film P3-T3 (the yellow dotted circles are the anatase); (c) schematic diagram of SiO2 strengthen by anatase and photo degradation mechanism, (CB, conduction band; VB, valence band). 
2017-04-03

\section{Robust transparent superamphiphobic coatings on non-fabric flat substrates with inorganic adhesive titania bonded silica}

Wen, Min

Royal Society of Chemistry

Wen M, Zhong J, Shuangjie Z, et al., Robust transparent superamphiphobic coatings on non-fabric flat substrates with inorganic adhesive titania bonded silica, Journal of Materials pÿChemistry A, Volume 5, Issue 18, Pages 83528359.

http://dx.doi.org/10.1039/C7TA01999H

Downloaded from Cranfield Library Services E-Repository 